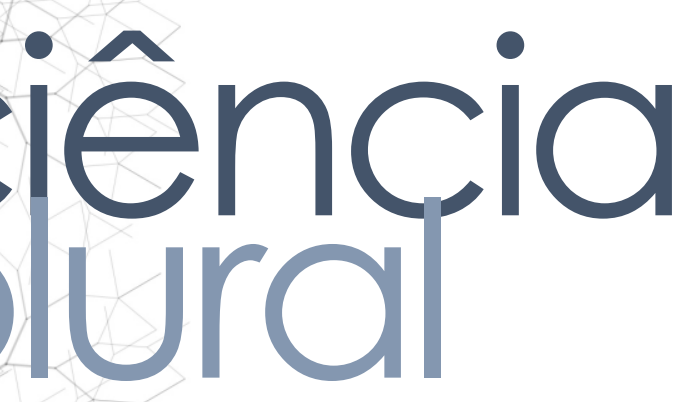

\title{
SEGURANÇA DO AMBIENTE DOMICILIAR E OCORRÊNCIA DE QUEDAS EM PESSOAS IDOSAS
}

The home environment safety and the occurrence of falls in elderly people

La seguridad en el entorno domiciliario y la ocurrencia de caídas en los ancianos

Zuleika Dantas do Vale Tavares • Enfermeira • Mestre em Saúde da Família pela Universidade Federal do Rio Grande do Norte - UFRN •

E-mail: zuleikaenfa@gmail.com

Mayara Priscilla Dantas Araújo • Nutricionista • Mestranda do Programa de Pós Graduação em Saúde Coletiva da UFRN • E-mail: mayaraaraujonutri@gmail.com

Vilani Medeiros de Araújo Nunes • Professora titular do Departamento de Saúde Coletiva da UFRN • Doutora em Ciências da Saúde pela UFRN •

E-mail: vilani.nunes@gmail.com

Autora correspondente:

Mayara Priscilla Dantas Araújo • E-mail: mayaraaraujonutri@gmail.com 


\section{ciênciála
pural}

\section{RESUMO}

Introdução: A ocorrência de quedas em pessoas idosas é considerada um problema de saúde pública, acarretando prejuízos para a saúde, mas que pode ser prevenida a partir da manutenção de um ambiente seguro. Objetivo: Identificar condições relacionadas a ocorrência de quedas e segurança do ambiente domiciliar de pessoas idosas residentes na zona rural de um município do Rio Grande do Norte. Metodologia: Foi realizada uma pesquisa-ação com uma das etapas de diagnóstico feito a partir de um estudo analítico e observacional. O estudo foi realizado com Agentes Comunitários de Saúde e idosos. Foram realizadas capacitações com os agentes para reconhecimento e identificação dos riscos ambientais de quedas nos domicílios dos idosos. Para coleta de dados, utilizou-se o questionário de avaliação ambiental e quedas da Caderneta de Saúde da Pessoa Idosa. Resultados: Participaram do estudo 288 idosos, dos quais $24,7 \%$ relataram ter sofrido queda e desses, 23,9\% tiveram fratura óssea. 47,9\% das quedas ocorreram dentro de casa, principalmente no banheiro (42,2\%). A maioria dos itens de segurança do ambiente domiciliar não estava adequado. Foram encontradas diferenças significativas entre a ocorrência de queda e a ausência de pisos uniformes e tapetes bem fixos $(p<0,001)$, interruptores acessíveis nas entradas de cômodos $(p<0,001)$, área do chuveiro com antiderrapante $(p=0,026)$ e armários baixos sem necessidade do uso de escadas $(\mathrm{p}<0,001)$. Conclusões: A maioria das pessoas idosas vive em um ambiente que favorece a ocorrência de quedas. Enfatiza-se a necessidade de ações de prevenção de quedas e segurança domiciliar tendo em vista suas implicações na qualidade de vida dessas pessoas.

Palavras-Chave: Idoso; Acidentes por Quedas; Habitação; Prevenção de Acidentes.

\section{ABSTRACT}

Introduction: The occurrence of falls in elderly people is considered a public health problem, causing harm to health, but this can be prevented by maintaining a safe environment. Objective: To identify conditions related to the occurrence of falls and home environment safety of elderly people living in the rural area of a city in Rio Grande do Norte, Brazil. Methodology: We performed an action-research with one of the diagnostic stages made from an analytical and observational study. The study was conducted with Community Health Workers and the local elderly population. Training was accomplished with these workers to recognize and identify the environmental risks of falls in the elderly's homes. In order to collect data, we used the environmental assessment and falls questionnaire from the Elderly Person's Health Booklet. Results: A total of 288 older adults participated in the study, of hom $24.7 \%$ reported having suffered a fall; and of these, $23.9 \%$ had a bone fracture. percentage of $47.9 \%$ of falls occurred indoors, mainly in the bathroom $(42.2 \%)$. ost home safety items were not adequate. Significant differences were found ween the occurrence of falls and the absence of uniform floors and well-fixed s $(p<0.001)$, accessible switches at the entrances of rooms $(p<0.001)$, non-slip wer area $(p=0.026)$ and low closets without the need to use stairs $(p<0.001)$. 
Conclusions: Most elderly people live in an environment that favors the occurrence of falls. Fall prevention and home safety actions are necessary due to their implications on the quality of life of this population.

Keywords: Aged; Accidental Falls; Housing; Accident Prevention.

\section{RESUMEN}

Introducción: La ocorruencia de caídas em los ancianos se considera un problema de salud pública, que causa daños a la salud, pero que puede prevenirse manteniendo un entorno seguro. Objetivo: Identificar las condiciones relacionadas con la ocurrencia de caídas y la seguridad em el entorno domiciliario de los ancianos que viven em um área rural de un municipio de Rio Grande do Norte, Brasil. Metodología: Se realizó una investigación-acción con una de las etapas de diagnóstico realizada a partir de un estudio analítico y observacional. El estudio se realizó con Agentes de Salud Comunitarios y ancianos. Los agentes recibieron formación para reconocer e identificar los riesgos ambientales de las caídas en las residencias de los ancianos. Para la recogida de datos, se utilizó el cuestionario de evaluación ambiental y caídas de la Cartilla de Salud de los Ancianos. Resultados: El estudio incluyó a 288 ancianos, de los cuales el 24,7\% sufrieron caída; y, de estos, el $23,9 \%$ tuvieron fractura ósea. El 47,9\% de las caídas se produjeron en un entorno interior, principalmente en el baño (el 42,2\%). La mayoría de los elementos de seguridad en el entorno domiciliario no eran adecuados. Se encontraron diferencias significativas entre la ocurrencia de caída y la ausencia de suelos uniformes y alfombras bien fijadas $(p<0,001)$, interruptores accesibles en las entradas de las habitaciones $(p<0,001)$, zona de ducha con antideslizante $(p=0,026)$ y armarios bajos sin necesidad de utilizar escaleras $(p<0,001)$. Conclusiones: Muchos ancianos viven en entornos que favorecen la ocurrencia de caídas. Las acciones de prevención de caídas y de seguridad em el hohar son necessárias por sus implicaciones en la calidad de vida de esta población.

Palabras clave: Anciano;; Accidentes por Caídas; Vivienda; Prevención de Accidentes. 


\section{ciêncíncia
pural}

\section{Introdução}

Com o aumento da população idosa ocorrem mudanças nas demandas de saúde e cuidados, uma vez que a senescência leva a alterações nos diferentes âmbitos da vida como também orgânicas. Uma dessas alterações, comumente observada em idosos, refere-se à redução da força muscular, que implica em mudanças na postura, modo de andar e equilíbrio, tornando a pessoa idosa mais susceptível as quedas ${ }^{1}$.

A ocorrência de quedas em pessoas idosas é considerada um problema de saúde pública e a principal causa externa de morbimortalidade nessa população ${ }^{2}$. As taxas de quedas entre essas pessoas variam de 28 a 35\% anualmente, no mundo, aumentando proporcionalmente com a idade ${ }^{3}$.

As quedas são caracterizadas por uma mudança não intencional do corpo, que sai da posição inicial para um nível inferior sem conseguir retornar à posição inicial em tempo hábil ${ }^{4}$. Sua ocorrência é decorrente de uma condição sindrômica multifatorial pela relação dos fatores intrínsecos, extrínsecos e comportamentais ${ }^{5}$.

Os fatores extrínsecos se dão pela interação do indivíduo com o ambiente em que vive, podendo contribuir para ocorrência de quedas a partir da exposição a riscos como ausência de barras de apoio e corrimões em escadas, pisos irregulares e escorregadios, iluminação inadequada, obstrução de áreas de locomoção, tapetes soltos, entre outros ${ }^{6}$. As quedas podem ser prevenidas com a mudança dos fatores de risco que são modificáveis e/ou evitáveis, garantindo assim um ambiente seguro para a pessoa idosa 5 .

A literatura sobre a avaliação e segurança do ambiente domiciliar para o risco de quedas em idosos é escassa. Muitos estudos abordam o sujeito que sofreu a queda, as consequências para a saúde e suas consequências na qualidade de vida após o agravo. Outros buscam compreender os fatores determinantes da queda a partir da sua ocorrência, porém são poucos os estudos que avaliam os fatores trínsecos como segurança do ambiente domiciliar para o risco de quedas, teriormente à ocorrência do evento queda. 
Diante disso, pelas consequências da ocorrência de quedas em pessoas idosas, como traumas, hospitalização e óbito, além de contribuir para o declínio funcional e diminuição da autonomia, que interferem diretamente na qualidade de vida dessas pessoas, torna-se necessária a prevenção da queda a partir do conhecimento dos fatores que levam a sua ocorrência, mais especificamente, no âmbito domiciliar.

Dessa forma, o presente estudo buscou identificar as condições relacionadas a ocorrência de quedas e segurança do ambiente domiciliar de idosos residentes na zona rural do município de São José de Mipibu, RN.

\section{Metodologia}

Trata-se de uma pesquisa-ação com uma das etapas de diagnóstico feita a partir de um estudo analítico e observacional com abordagem quantitativa. A pesquisa foi realizada com Agentes Comunitários de Saúde (ACS) e idosos residentes na zona rural do município de São José de Mipibu, localizado na Região Metropolitana de Natal, Estado do Rio Grande do Norte.

Como estratégia de ação, foi elaborado um guia de prevenção de quedas para auxiliar na capacitação dos ACS voltada para o reconhecimento dos riscos extrínsecos e aspectos ligados à sua prevenção. O referido guia aborda questões relacionadas ao ambiente, fornecendo orientações para prevenção de quedas em diferentes locais do domicílio, além de instruções para realização de atividades de vida diária de forma segura.

Foram realizadas duas oficinas de capacitação com os ACS com carga horária de oito horas cada, nos dias 02 e 03 de fevereiro de 2018, com a participação da nutricionista e fisioterapeuta do Programa Academia da Saúde do município.

A $1^{\mathrm{a}}$ oficina foi direcionada para orientações de utilização do guia, sendo realizada leitura compartilha e discussão sobre o tema. A $2^{\mathrm{a}}$ oficina foi destinada a orientação dos ACS quanto ao preenchimento correto da Caderneta de Saúde da ssoa Idosa, instrumento de coleta de dados utilizado nessa pesquisa. 
Para os idosos, foi utilizada uma amostra probabilística aleatória simples. O cálculo se deu com base na população de idosos da zona rural do município, que consistia em 2.271 idosos 7 . Foi realizado o cálculo para o tamanho da amostra $(n)$ de forma casual simples, que resultou em 283 sujeitos. Foram incluídos no estudo pessoas idosas (a partir de 60 anos), de ambos os sexos, assistidos por alguma das equipes de Saúde da Família da zona rural do município.

Os dados foram obtidos a partir da Caderneta de Saúde da Pessoa Idosa (CSPI), versão 2014, preenchidas pelos ACS em visita domiciliar. Esse instrumento permite o acompanhamento das condições de saúde da pessoa idosa, incluindo a ocorrência de quedas e avaliação ambiental, podendo ser utilizado de forma estratégica para ações de prevenção de quedas em pessoas idosas ${ }^{8}$.

Além dos dados da CSPI, como forma de complementar as informações, foram incluídas as seguintes questões sobre a ocorrência de quedas no instrumento: Para a questão: qual foi o local da queda? Dentro de casa (onde?) Ou fora de casa (onde?).

Como o município ainda não havia realizado a implantação do uso da caderneta entre os idosos, cada um dos 28 ACS participantes recebeu 15 CSPI para serem distribuídas entre os idosos nas visitas domiciliares, que foram escolhidos de forma aleatória para participação no estudo. A coleta de dados se deu entre os meses de fevereiro e abril de 2018.

Os dados coletados foram tabulados no programa Excel versão 2010. Para realização das análises estatísticas foi utilizado o software Statistical Package for the Social Sciences (SPSS), versão 21.0. Foram realizadas análises descritivas e o teste quiquadrado, considerando o nível de significância inferior a 0,05 e intervalo de confiança de $95 \%$.

O estudo foi aprovado pelo Comitê de Ética do Hospital Universitário Onofre Lopes (CEP-HUOL) sob o número 1.375.353, segundo preconizado pela Resolução n ${ }^{\circ}$ 6/ 2012 do Conselho Nacional de Saúde. 


\section{Resultados}

Foram coletados dados de 288 idosos residentes na zona rural do município de São José de Mipibu. Destes, 156 (54,2\%) eram do sexo feminino, 167 (58,0\%) tinham até 70 anos, 157 (54,5\%) afirmaram ser casados, 208 (72,2\%) eram católicos, $154(53,5 \%)$ sabiam ler e escrever, embora apenas 148 (48,6\%) tivessem estudo formal e $248(86,1 \%)$ afirmaram receber aposentadoria ou pensão. A principal ocupação foi a agricultura $(50,0 \%)$, seguida por atividades domésticas (26,7\%). A maior parte dos idosos (89,6\%) afirmaram não possuir qualquer tipo de dificuldade auditiva, visual, intelectual/cognitiva, física ou outra.

Ao avaliar a associação entre a ocorrência de quedas e com quem reside, não foi encontrada associação estatisticamente significante $(p=0,127)$. É possível observar que a maior parte dos idosos mora com os companheiros e familiares $(44,1 \%)$. A ocorrência de quedas foi maior naqueles que residem com familiares $(34,2 \%)$ e a não ocorrência foi maior naqueles que residem apenas com o companheiro $(83,0 \%)$ (Tabela 1).

Tabela 1. Associação entre a ocorrência de queda e com quem residem os idosos na zona rural do município de São José de Mipibu, Rio Grande do Norte. Natal-RN, Brasil, 2018.

\begin{tabular}{lccccccc}
\hline \multirow{2}{*}{ Com quem reside } & \multicolumn{4}{c}{ Ocorrência de queda } & \multicolumn{2}{c}{ Total } & \multirow{2}{*}{ p-valor $^{1}$} \\
\cline { 2 - 7 } & $\mathrm{n}$ & $\%$ & $\mathrm{n}$ & $\%$ & $\mathrm{n}$ & $\%$ & \\
\hline Com companheiro & 8 & 17,0 & 39 & 83,0 & 47 & 16,3 & \\
Com familiares & 26 & 34,2 & 50 & 65,8 & 76 & 26,4 & \multirow{2}{*}{0,127} \\
Familiares/Companheiro & 28 & 22,1 & 99 & 78,0 & 127 & 44,1 & \\
Sozinho & 9 & 23,7 & 76,3 & 38 & 38 & 13,2 & \\
\hline
\end{tabular}

${ }^{1}$ Teste qui-quadrado.

Na tabela 2 observam-se os itens para segurança do domicílio separados por ambiente. Quanto à segurança nas áreas de locomoção, 154 (53,5\%) idosos relataram hpedimento nessas áreas e 160 (55,6\%) relataram não ter pisos uniformes e tapetes os. No banheiro, apenas $78(27,1 \%)$ tem antiderrapante na área do chuveiro e 212 6\%) não tem box com abertura fácil ou presença de cortina firme. Quanto a 
escada, 42 (14,6\%) idosos afirmaram possuir escada no domicílio, porém, nenhuma apresenta corrimão dos dois lados e firmes e/ou piso antiderrapante.

Tabela 2. Segurança do domicílio de idosos residentes na zona rural do município de São José de Mipibu, Rio Grande do Norte. Natal-RN, Brasil, 2018.

\begin{tabular}{llccc}
\hline & \multicolumn{1}{c}{ Itens } & & $\mathbf{n}$ & \% \\
\hline Áreas de & Áreas de locomoção desimpedidas & Sim & 134 & 46,5 \\
locomoção & & Não & 154 & 53,5 \\
& Presença de barra de apoio & Sim & 27 & 9,4 \\
& & Não & 261 & 90,6 \\
& Pisos uniformes e tapetes bem fixos & Sim & 128 & 44,4 \\
Iluminação & & Não & 160 & 55,6 \\
& Presença de luzes suficientes para todo & Sim & 255 & 88,5 \\
& interior dos cômodos e degraus & Não & 33 & 11,5 \\
& Interruptores acessíveis nas entradas de & Sim & 208 & 72,2 \\
Banheiro & cômodos & Não & 80 & 27,8 \\
& Área do chuveiro com antiderrapante & Sim & 78 & 27,1 \\
& & Não & 210 & 72,9 \\
& Box com abertura fácil ou presença de cortina & Sim & 76 & 26,4 \\
Cozinha e e & Não & 212 & 73,6 \\
quarto & Armários baixos sem necessidade do uso de & Sim & 213 & 74,0 \\
Escada & escadas & Não & 75 & 26,0 \\
& Possuem escadas & Sim & 42 & 14,6 \\
& & Não & 246 & 85,4 \\
& Corrimão dos dois lados e firmes. Piso & Sim & 0 & 0,0 \\
& antiderrapante & Não & 288 & 100,0 \\
\hline
\end{tabular}

Dentre os idosos que participaram do estudo, 71 (24,7\%) afirmaram já ter sofrido alguma queda. Quanto ao local, a maioria ocorreu dentro de casa $(47,9 \%)$, seguida de fora de casa $(36,2 \%)$ e dentro e fora de casa $(15,5 \%)$. Esse último evidencia que alguns idosos caíram mais de uma vez. Dentro de casa, o local de maior ocorrência de quedas foi o banheiro $(42,2 \%)$. Fora de casa, as quedas no quintal foram as mais frequentes $(51,4 \%)$ (Tabela 3$)$.

A ocorrência de queda levou a algum tipo de fratura em 17 (23,9\%) idosos. A ratura da bacia foi a mais frequente (23,5\%). Após a queda, $15(21,1 \%)$ idosos irmaram ter paralisado suas atividades por medo (Tabela 3 ). 
Tabela 3. Ocorrência de queda em idosos residentes na zona rural do município de São José de Mipibu, Rio Grande do Norte. Natal-RN, Brasil, 2018.

\begin{tabular}{|c|c|c|c|}
\hline \multicolumn{2}{|l|}{ Ocorrência de queda } & \multirow{2}{*}{$\begin{array}{l}\mathbf{n}^{\mathbf{1}} \\
71\end{array}$} & \multirow{2}{*}{$\begin{array}{c}\% \\
24,7\end{array}$} \\
\hline Ocorrência de queda & Sim & & \\
\hline & Não & 217 & 75,3 \\
\hline \multirow[t]{3}{*}{ Local da queda } & Dentro de casa & 34 & 47,9 \\
\hline & Fora de casa & 26 & 36,6 \\
\hline & Dentro e fora de casa & 11 & 15,5 \\
\hline \multirow[t]{5}{*}{ Local da queda dentro de casa ${ }^{1}$} & Banheiro & 19 & 42,2 \\
\hline & Sala & 11 & 24,4 \\
\hline & Quarto & 10 & 22,2 \\
\hline & Cozinha & 6 & 13,3 \\
\hline & Área de serviço & 1 & 2,2 \\
\hline \multirow[t]{3}{*}{ Local da queda fora de casa ${ }^{1}$} & Quintal & 19 & 51,4 \\
\hline & Rua & 16 & 43,2 \\
\hline & Roçado & 2 & 5,4 \\
\hline \multirow[t]{2}{*}{ Fratura decorrente da queda } & Sim & 17 & 23,9 \\
\hline & Não & 54 & 76,1 \\
\hline \multirow[t]{7}{*}{ Local da fratura } & Bacia & 4 & 23,5 \\
\hline & Braço & 3 & 17,7 \\
\hline & Cabeça & 3 & 17,7 \\
\hline & Perna & 2 & 11,8 \\
\hline & Costela & 1 & 5,9 \\
\hline & Mão & 1 & 5,9 \\
\hline & Não lembra o local & 3 & 17,7 \\
\hline \multirow[t]{2}{*}{ Paralisação de atividades por medo } & Sim & 15 & 21,1 \\
\hline & Não & 56 & 78,9 \\
\hline
\end{tabular}

${ }^{1}$ Variação no $n$ decorrente da possibilidade de múltipla resposta.

A Tabela 4 mostra a associação entre a ocorrência de queda e segurança do domicílio dos idosos avaliados. Foram encontradas diferenças estatisticamente significativas entre a ocorrência de queda e a não conformidade dos itens: pisos uniformes e tapetes bem fixos $(p<0,001)$, interruptores acessíveis nas entradas de cômodos $(p<0,001)$, área do chuveiro com antiderrapante $(p=0,026)$ e armários baixos mecessidade do uso de escadas $(p<0,001)$. 
Tabela 4. Associação entre a ocorrência de queda e a segurança do domicílio de idosos residentes na zona rural do município de São José de Mipibu, Rio Grande do Norte. Natal-RN, Brasil, 2018.

\begin{tabular}{|c|c|c|c|c|c|c|c|c|}
\hline \multirow{3}{*}{ Segurança do ambiente } & & \multicolumn{4}{|c|}{ Ocorrência de queda } & \multirow{2}{*}{\multicolumn{2}{|c|}{ Total }} & \multirow{3}{*}{$\mathrm{p}$-valor ${ }^{1}$} \\
\hline & & \multicolumn{2}{|c|}{ Sim } & \multicolumn{2}{|c|}{ Não } & & & \\
\hline & & $\mathrm{n}$ & $\%$ & $\mathrm{n}$ & $\%$ & $\mathrm{~N}$ & $\%$ & \\
\hline \multirow{2}{*}{$\begin{array}{l}\text { Áreas de locomoção } \\
\text { desimpedidas }\end{array}$} & Sim & 26 & 19,4 & 108 & 80,6 & 134 & 46,5 & \multirow{2}{*}{0,054} \\
\hline & Não & 45 & 29,2 & 109 & 70,8 & 154 & 53,5 & \\
\hline \multirow{2}{*}{$\begin{array}{l}\text { Presença de barra de } \\
\text { apoio }\end{array}$} & Sim & 5 & 18,5 & 22 & 81,5 & 27 & 9,4 & \multirow{2}{*}{0,437} \\
\hline & Não & 66 & 25,3 & 195 & 74,7 & 261 & 90,6 & \\
\hline \multirow{2}{*}{$\begin{array}{l}\text { Pisos uniformes e } \\
\text { tapetes bem fixos }\end{array}$} & Sim & 15 & 11,7 & 113 & 88,3 & 128 & 44,4 & \multirow{2}{*}{0,000} \\
\hline & Não & 56 & 35,0 & 104 & 65,0 & 160 & 55,6 & \\
\hline \multirow{2}{*}{$\begin{array}{l}\text { Presença de } \\
\text { iluminação suficiente } \\
\text { para todo interior dos } \\
\text { cômodos e degraus }\end{array}$} & Sim & 62 & 24,3 & 193 & 75,6 & 255 & 88,5 & \multirow[b]{2}{*}{0,711} \\
\hline & Não & 9 & 27,3 & 24 & 72,7 & 33 & 11,5 & \\
\hline \multirow{4}{*}{$\begin{array}{l}\text { Interruptores } \\
\text { acessíveis nas } \\
\text { entradas de cômodos } \\
\text { Área do chuveiro } \\
\text { com antiderrapante }\end{array}$} & Sim & 32 & 15,4 & 176 & 84,6 & 208 & 72,2 & \multirow{2}{*}{0,000} \\
\hline & Não & 39 & 48,7 & 41 & 51,3 & 80 & 27,8 & \\
\hline & Sim & 12 & 15,4 & 66 & 84,6 & 78 & 27,1 & \multirow{2}{*}{0,026} \\
\hline & Não & 59 & 28,1 & 151 & 71,9 & 210 & 72,9 & \\
\hline \multirow{2}{*}{$\begin{array}{l}\text { Box com abertura } \\
\text { fácil ou presença de } \\
\text { cortina firme }\end{array}$} & Sim & 14 & 18,4 & 62 & 81,6 & 76 & 26,4 & \multirow[b]{2}{*}{0,142} \\
\hline & Não & 57 & 26,9 & 155 & 73,1 & 212 & 73,6 & \\
\hline \multirow{2}{*}{$\begin{array}{l}\text { Armários baixos sem } \\
\text { necessidade do uso } \\
\text { de escadas }\end{array}$} & Sim & 34 & 16,0 & 179 & 84,0 & 213 & 74,0 & \multirow{2}{*}{0,000} \\
\hline & Não & 37 & 49,3 & 38 & 50,7 & 75 & 26,0 & \\
\hline \multirow[t]{2}{*}{ Piso antiderrapante } & Sim & 8 & 19,0 & 34 & 81,0 & 42 & 14,6 & \multirow{2}{*}{0,362} \\
\hline & Não & 63 & 25,6 & 183 & 74,4 & 246 & 85,4 & \\
\hline
\end{tabular}

1 Teste qui-quadrado.

\section{Discussão}

Ao analisar as características demográficas da amostra, verificou-se maior revalência de pessoas idosas do sexo feminino, com idade até 70 anos, casadas, atólicas, que sabiam ler e escrever e recebiam aposentadoria ou pensão. Tais ados corroboram com o observado na literatura por outros estudos, 
Em estudo de revisão sistemática realizado por Sousa et al., ${ }^{3}$ foi identificado que o sexo feminino, a baixa escolaridade e o baixo rendimento familiar são fatores de risco para quedas em pessoas idosas. Apesar disso, as pessoas idosas avaliadas não apresentaram alguns dos fatores intrínsecos que contribuem para a ocorrência de quedas, como a idade avançada, déficit cognitivo e diminuição da acuidade visual e auditiva, que levam a uma menor capacidade de identificar e evitar obstáculos, além da lentidão dos reflexos decorrentes da própria senescência2,5,12.

Quanto a ocorrência de quedas, foi encontrado no presente estudo prevalência de 24,7\%. Estudo realizado por Araújo et al., ${ }^{4}$ encontrou igual prevalência ao avaliar idosos residentes na zona urbana de Minas Gerais. Apesar disso, a literatura traz valores mais elevados, variando entre $37,7 \%$ e $64,5 \%$ a prevalência de quedas em idosos $5,13,14$. A menor prevalência em comparação à literatura é um achado positivo, uma vez que a ocorrência de quedas é a principal causa externa de morbimortalidade em idosos ${ }^{2}$.

Embora não tenha sido encontrada associação entre a ocorrência de queda e residir sozinho ou acompanhado, seu acontecimento foi mais frequentes naqueles que residiam com familiares. Segundo Alves et al.,15 isso pode ser explicado pelo protecionismo da família, que pode considerar a pessoa idosa incapaz de realizar algumas atividades e acabar por realizá-las como forma de zelo e cuidado, contribuindo para maior dependência e perda de autonomia dessas pessoas, o que pode aumentar a probabilidade de queda.

Em relação ao local de ocorrência, a maioria se deu dentro do próprio domicílio, principal cenário das quedas ${ }^{2}$, o que demonstra a necessidade de manter esse ambiente seguro a fim de evitar as quedas e os consequentes prejuízos à saúde da pessoa idosa. Dentro do domicílio, o banheiro foi o cômodo com maior frequência de quedas. Fora do domicílio, as quedas se deram principalmente no quintal. Esses achados são corroborados por outros estudos $2,4,15$.

A ocorrência de quedas nesses espaços pode estar relacionada a insegurança ambiente, a qual foi analisada por esse estudo e que pode explicar este achado. itos idosos vivem em ambientes que não apresentam segurança contra quedas, 
principalmente banheiro e escadas, quando presentes. Além disso, foi encontrada associação entre a ocorrência de queda e ausência de pisos uniformes e tapetes fixos, interruptores acessíveis nas entradas de cômodos, área do chuveiro com antiderrapante e armários baixos sem necessidade do uso de escadas.

Em estudo realizado por Silva, Rezende e Calábria, 14 41,4\% (n=24) dos idosos analisados declararam ter risco aparente para queda em sua casa, dentre eles a presença de escadas, degraus e piso escorregadios. A não conformidade de itens de segurança contribui para a ocorrência de quedas no ambiente domiciliar como mostra o achado do presente estudo.

Como forma de tornar o ambiente domiciliar mais seguro, orienta-se para o uso de piso antiderrapante, plano e regular, tapetes fixos, adequação da iluminação dos cômodos, colocação de barras de apoio e corrimão ao lado das escadas, tapetes antiderrapante na área do chuveiro, manter as áreas de locomoção desimpedidas 4 .

Estudo realizado em São Paulo analisou 261 idosos domiciliados e encontrou prevalência de risco de queda de $51,7 \%$, que pode estar relacionado tanto a fatores intrínsecos quanto extrínsecos ${ }^{1}$. Isso mostra que a prevenção de quedas não se restringe a cuidados de saúde, mas também ao cuidado do ambiente, tornando-o seguro e adequado às necessidades da pessoa idosa.

A manutenção de um ambiente seguro contribui para evitar as quedas, uma vez que elas podem levar a desfechos adversos a saúde como lesões, fraturas ósseas, hospitalização e morte ${ }^{1}$. 23,9\% dos idosos avaliados sofreram alguma fratura em decorrência da queda. Alves et al.,15 em estudo realizado com 206 idosos adscritos a uma equipe da Estratégia da Saúde da Família (ESF) do município de Barbacena, Minas Gerais, relatou a ocorrência de fratura em 8,7\% dos idosos e que, após a queda, 29,3\% dos idosos apresentaram dificuldades para realizar atividades diárias e $17,3 \%$ pararam de realizá-las. Resultado semelhante foi encontrado em estudo de base populacional realizado por Pimentel et al. ${ }^{10}$ quanto a fratura de quadril e fêmur idosos, que apresentou frequência de 8,3\%, sendo necessária intervenção úrgica em 44,3\% dos casos. 


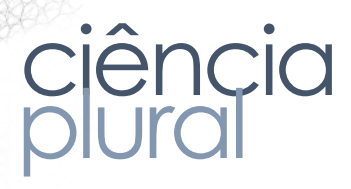

Além das consequências físicas, as quedas podem afetar a saúde mental do idoso, levando ao medo de cair novamente e até mesmo a não realização das atividades diárias. Dentre as consequências observadas após a queda em idosos estão o medo de cair novamente, a dependência para realização de atividades de vida diária, a perda da autonomia e depressão/isolamento ${ }^{4}$. A garantia de um ambiente seguro a fim de evitar a ocorrência de quedas, pode contribuir para a qualidade de vida e manutenção da independência e autonomia da pessoa idosa.

Como limitação do estudo, pontua-se a abordagem somente dos fatores extrínsecos, embora o risco de quedas também esteja relacionado aos fatores intrínsecos, como uso de medicamentos, a presença de doenças crônicas, síndrome da fragilidade, entre outros. Essas informações podem ser analisadas em estudos futuros de forma a compreender a multiplicidade dos fatores de risco para quedas em idosos.

A maioria das pessoas idosas vive em um ambiente que favorece a ocorrência de quedas, sendo observada a associação entre o seu acontecimento e não conformidade de alguns itens de segurança, com destaque para o banheiro, cômodo onde ocorreu a maior parte das quedas.

A queda pode ocasionar desfechos adversos à saúde, levando a uma maior demanda de atenção e gastos em saúde, sendo considerada um problema de saúde pública. Com isso, e pelo rápido envelhecimento populacional, há uma maior necessidade de ações de prevenção de quedas e segurança domiciliar para pessoas idosas.

Destaca-se a importância da atuação dos ACS na identificação dos riscos presentes no ambiente domiciliar e orientação dos usuários acerca das medidas de segurança necessárias para evitar as quedas durante as visitas domiciliares, assim omo a importância da utilização da CSPI como forma de monitorar a ocorrência de uedas e o ambiente, como também as condições de saúde. 


\section{Conclusões}

Os resultados do estudo mostram que os idosos que residem na zona rural do município de São José de Mipibu podem estar mais propensos a ocorrência de quedas devido a não conformidade de itens de segurança do ambiente domiciliar. Os itens que levaram a uma maior ocorrência de quedas foram a presença de pisos desnivelados, tapetes soltos, interruptores inacessíveis nas entradas de cômodos, falta de antiderrapante na área do chuveiro e armários altos.

O reconhecimento das inadequações ambientais que levam a maior ocorrência de quedas pelos ACS, assim como sua capacitação para orientação e intervenção junto a pessoa idosa e seus familiares é de fundamental importância para prevenção desse evento, que traz prejuízos diretos para qualidade de vida desses indivíduos.

\section{Referências}

1. Giacomini SBL, Fhon JR, Rodrigues RAP. Fragilidade e risco de queda em idosos que vivem no domicílio. Acta Paul Enferm. 2020 Jun;33:1-8. Disponível em: http://dx.doi.org/10.37689/acta-ape/2020AO0124

2. Leitão SM, Oliveira SC, Rolim LR, Carvalho RP, Coelho Filho JM, Peixoto Junior AA. Epidemiologia das quedas entre idosos no Brasil: uma revisão integrativa de literatura. Geriatr Gerontol Aging. 2018 Set;12(3):172-9. Disponível em: http:/ /dx.doi.org/10.5327/Z2447-211520181800030

3. Sousa LMM, Marques-Vieira CMA, Caldevilla MNGN, Henriques CMAD, Severino SSP, Caldeira SMA. Risco de quedas em idosos residentes na comunidade: revisão sistemática da literatura. Rev Gaúcha Enferm. 2016 Fev;37(4):e55030. Disponível em: http://dx.doi.org/10.1590/19831447.2016.04.55030

Araújo IVS, Gomes NC, Nascimento JS, Ribeiro CCNR, Tavares DMDS. Queda entre idosos: preditores e distribuição espacial. Rev Salud Pública. 2019 Mar;21(2):187-94. Disponível em: https://doi.org/10.15446/rsap.V21n2.70298

Chehuen Neto JA, Brum IV, Braga NAC, Gomes GF, Tavares PL, Silva RTC, et al. Percepção sobre queda como fator determinante desse evento entre idosos residentes na comunidade. Geriatr Gerontol Aging. 2017;11(1):25-31.

Teixeira DKS, Andrade LM, Santos JLP, Caires ES. Quedas em pessoas idosas: 
restrições do ambiente doméstico e perdas funcionais. Rev Bras Geriatr e Gerontol. 2019 Set;22(3):1-10. Disponível em: https://doi.org/10.1590/198122562019022.180229

7. Instituto Brasileiro de Geografia e Estatística (IBGE). Censo demográfico 2010. Rio de Janeiro: IBGE; 2012.

8. Brasil. Ministério da Saúde. Caderneta de Saúde da Pessoa Idosa. Brasília: Ministério da Saúde; 2014.

9. Silva IGP, Peruzzo HE, Lino IGT, Marquete VF, Marcon SS. Perfil sociodemográfico e clínico de idosos em risco de quedas no sul do Brasil. J Nurs Heal. 2019;9(3). Disponível em: https://doi.org/10.15210/jonah.v9i3.16808

10. Pimentel WRT, Pagotto V, Stopa SR, Hoffmann MCCL, Malta DC, Menezes RL. Quedas com necessidade de procura de serviços de saúde entre idosos: uma análise da Pesquisa Nacional de Saúde, 2013. Cad Saúde Pública. 2018 Ago;34(8). Disponível em: http://dx.doi.org/10.1590/0102-311x00211417

11. Santos F, Lange C, Llano PMP, Lemões MAM, Pastore CA, Paskulin LMG, et al. Quedas de idosos residentes na zona rural: prevalência e fatores associados. Rev Bras Enferm. 2019;72(Suppl 2):4-6. Disponível em: http://dx.doi.org/10.1590/0034-7167-2018-0460

12. Smith AA, Silva AO, Rodrigues RAP, Moreira MASP, Nogueira JA, Tura LFR. Avaliação do risco de quedas em idosos residentes em domicílio. Rev Lat Am Enfermagem. 2017 Abr;25. Disponível em: http:// dx.doi.org/10.1590/15188345.0671.2754

13. Oliveira $\mathrm{T}$, Baixinho CL, Henriques MA. Risco multidimensional de queda em idosos. Rev Bras Promoç Saúde. 2018 Abr/Jun;31(2):1-9. Disponível em: http://dx.doi.org/10.5020/18061230.2018.7058

14. Silva EO, Rezende AAA, Calábria LK. Aspectos socioeconômicos e eventos de queda entre idosos atendidos no sistema público de saúde. Rev Bras Promoç Saúde. 2019;32:1-9. Disponível em: http:// dx.doi.org/10.5020/18061230.2019.9532

15. Alves RLT, Silva CFM, Pimentel LN, Costa IA, Souza ACS, Coelho LAF. Avaliação dos fatores de risco que contribuem para queda em idosos. Rev Bras Geriatr e Gerontol. 2017 Fev;20(1):56-66. Disponível em: https://doi.org/10.1590/1981-22562017020.160022 